\title{
X-RAY AND GAMMA-RAY POLARIMETRY OF GRBS
}

\author{
E. Costa ${ }^{1}$
}

\begin{abstract}
Polarimetry is expected to play a major role as a diagnostic tool for GRBs. Techniques and methods for X/Gamma ray polarimetry are reviewed including the specific problems related to the transient nature of the sources. Optical data do not encourage optimistic predictions on polarimetry of afterglows. I review some of the existing and proposed experiments for the prompt and discuss the existing results.
\end{abstract}

\section{Introduction}

Polarimetry is a powerful diagnostic tool in X-ray and Gamma-ray astronomy in more than one astrophysical context. Polarization may be intrinsic to the emission process, or introduced by the transfer of the radiation from the source to the observer, when the geometry highly deviates from spherical symmetry. GRB show non thermal spectra, where synchrotron likely plays an important role and evolve so fast that thermalization is unlikely. They could be beamed or, in any case, the so called relativistic collimation introduces a selection of emitting regions visible to the observer, preventing the large scale smearing of polarization. Therefore GRB have been candidate for polarimetry but measuring the polarization of $\mathrm{X}$ and Gama rays is not easy and needs dedicated instruments and observing strategies. $\mathrm{X}$-ray polarimetry of afterglows is only a special case of the polarimetry of X-ray sources, that has not been attempted any more since 35 years. The polarimetry of the prompt emission has been performed as a byproduct of instruments, built for other purposes. The very first data from a dedicated instrument are now coming. I discuss the validity and the significance of these data.

\section{How can we measure the polarization of hard $X$-rays and soft Gamma-rays}

Every polarimeter is composed of an analyzer, where an interaction occurs, whose outcome angle depends on polarization, and a detector of the products of the

1 Istituto di Astrofisica e Planetologia Spaziali, 031 INAF, via del Fosso del Cavaliere, 100, Roma, Italy 
interaction, to measure their angular distribution. Polarimeters can be dispersive when each angle is sampled at each time and not dispersive where all the angles are measured simultaneously. Dispersive polarimeters require the rotation around the optical axis. Non dispersive polarimeters in principle do not need, but in most cases the instrument response is not symmetric around the axis and rotation is needed to compensate the systematic effects. Every measurement of polarization can be reduced to building a modulation curve, namely a histogram of counts as a function of the angle. If the beam is not polarized the curve is flat:

$$
N(\varphi)=\text { constant }
$$

if it is polarized to some degree the modulation curve can be represented with the equation

$$
N(\varphi)=A+B \cos ^{2}(\varphi)
$$

where the constant term A accounts for both the unpolarized fraction and the non ideal performance of the device. We can therefore define the modulation as:

$$
M=\frac{N_{\max }-N_{\min }}{N_{\max }+N_{\min }} .
$$

With a beam $100 \%$ polarized the modulation depends only on the instrument and is named $\mu$ or modulation factor. Thence the measurement of the polarization is:

$$
\Pi=\frac{1}{\mu} \times \frac{B}{B+2 A} .
$$

The Equation (2.1) is in practice a series of counts distributed with poisson statistics around the expected values. The constant B in 2.2 can be seen as the second term of the power spectrum in the fourier expansion of this series. $\Pi$ is a positively defined quantity following a $\chi^{2}$ distribution with 2 d.o.f.. By integrating we can compute the so called Minimum Detectable Polarization, conventionally given for a $99 \%$ probability.

$$
M D P=\frac{4.29}{\varepsilon \mu F} \times \sqrt{\frac{B+\varepsilon F}{S T}}
$$

where, $\mathrm{S}$ is the collecting surface of the instrument, $\varepsilon$ is the efficiency, B is the background for unit surface and $\mathrm{T}$ the observing time.

This gives what we need, in terms of flux, area and observing time, to reject the hypothesis that a source is unpolarized. For a measurement of the polarization at $3 \sigma$ level the time required is around twice that needed for the MDP (Weisskopf et al. 2009).

\section{$2.1 X$-rays}

The Bragg diffraction at $45^{\circ}$ was the process applied for polarimetry in the first 25 years of X-ray Astronomy. The device is dispersive and the crystal and the 
detector (mounted at $90^{\circ}$ from the optical axis) are rotated around the axis (historically the whole satellite was rotating). The modulation factor is 1 but the band width is limited to few $\mathrm{eV}$ and the background high, so that the measurement is reliable but the sensitivity is poor. This can be mitigated by using mosaic crystals, with a broader rocking curve, slightly bent to achieve a moderate focussing. This method was applied aboard OSO-8 for the only positive detection of polarization ever in the X-ray band (Weisskopf et al. 1976). In a telescope, a small flat crystal can be mounted at $45^{\circ}$ before the focus and the photons, compliant with the Bragg condition, are focussed on a secondary plane in a newtonian mounting.

Around 10 years ago a big change occurred with the introduction of detectors based on photoelectric effect within a gas. The photoelectrons ejected from the K-shell (the large majority) have angular distribution of $\cos ^{2}$ around the electric vector. This means that, were we able to measure this direction, the process would be a perfect analyzer of linear polarization. This was well known since the beginning of X-ray astronomy. The troubles derive from the fact that, in the range of $\mathrm{X}$-rays, the electrons propagate in materials much less than photons of the equivalent energy. The newly produced electron ionizes the material and eventually is completely stopped. But it scatters as well, so that the information on the direction is quickly lost. To derive this direction one must be able to analyze the charge distribution within a layer of material which is a very small fraction of the thickness of material needed to stop a photon with a decent efficiency. As a consequence the method could be efficiently applied only with gas detectors and only after the progress in microelectronics allowed for the development of detectors finely subdivided. The first implementation was in Costa et al. (2001). A gas cell is polarized with a drift field parallel to the optical axis. The electrons of the track created in the gas by the photoelectron, are drifted by a constant field to a Gas Electron Multiplier, that amplifies the track while preserving the shape and the proportionality to the charge. The amplified track is collected by a read out plane finely pixellated. A further improvement came soon after (Bellazzini et al. 2004) when the readout plane was built as the top of an ASIC VLSI chip. The effect is that a single solid block includes the bottom of the detector, the read-out pad, an individual analog electronic chain for each of the 100000 pads, a triggering system and a pre-selection electronics that allows to fetch out the content of only a frame around pixels that passed the threshold. The content of these pixels is A/D converted. From the analysis of the track the impact point, the direction angle and the total charge are derived. In the focus of a telescope this device, named Gas Pixel Detector can therefore perform at the same time images, spectroscopy, timing and polarization.

A different concept to exploit the same physical process was developed, a few years later, at GSFC (Black 2007). In a gas cell an electric field, perpendicular to the optical axis, drifts the electrons of the track to a GEM and then to a set of strips that make the one-dimensional projection of the track. The image in the drift direction is performed with the method of the Time Projection Chamber. This method looses the information on the absorption point and needs rotation because systematics heavily depend on the drift direction. The GPD polarimeter 
is truly imaging, needs no rotation and minimizes background and systematics, while the TPC makes polarimetry of everything falls within a large field of view and needs rotation, but can be built thicker and, thence, more efficient.

The role of diffraction is not completely disappeared. Herman L. Marshall at MIT developed an instrument based on diffraction from multilayered artificial crystals (Marshall 2010) of photons collected by a telescope. The method is effective at very low energies.

\subsection{Gamma-rays}

The application of the photoelectric effect in gases in principle could be extended up to xenon but, in practice has not, due to the higher scattering/stopping ratio and to the highly not-diagonal response matrix. The technique of the photoelectron in gas has been so far limited to mixtures based on pressurized Argon, which restricts the application below $30 \mathrm{keV}$.

At higher energies the dominant interaction is the Compton effect. If we express $\mathrm{E}$ and $\mathrm{E}^{\prime}$ the energy of the photon before and after the scattering respectively, the Klein Nishina cross section is

$$
\frac{d \sigma}{d \Omega}=\frac{r_{0}^{2}}{2} \frac{E^{\prime 2}}{E^{2}}\left(\frac{E}{E^{\prime}}+\frac{E^{\prime}}{E}-2 \sin ^{2} \vartheta \cos ^{2} \varphi\right)
$$

where $r_{0}$ is the classic radius of the electron, $\vartheta$ is the scattering angle and $\varphi$ the azimuth scattering angle. Contrary to the photoelectric effect the modulation depends on the angle of scattering and decreases with the energy. Around $90^{\circ}$ and at low energies the scattering is an almost ideal analyzer, while the forward and back scattering loose memory of polarization. A polarimeter based on this effect is always composed of one or more scatterer and one or more absorber, which is always a detector. They may be passive when the scatterer is an inert material of low $\mathrm{Z}$ ( $\mathrm{Li}$ or $\mathrm{Be}$ ) or active, when the scatterer is a detector itself, in coincidence with the absorber(s). The first are cheaper and easier to do but the background is high and the sensitivity is good for strong sources only.

Active polarimeters can be also focal plane instruments in the focus of multilayer optics (Krawczynski et al. 2011; Fabiani et al. 2013).

The most studied configuration, without optics (McConnell 2010), is based on an array of detectors, to achieve a large collecting area. The radiation scattered on one detector is absorbed in another one and the time coincidence identifies the pair. To each pair of detectors corresponds a projected angle of the scattered photon. If the direction of the impinging photon is known the scattering angle is derived and the histogram (modulation curve) built. Two different concepts are there:

- one-phase. The scatterer and the absorber are detectors of the same material.

- two-phases. The array includes detectors of different atomic number. Typically organic (low Z) scintillator to act as a scatterer and inorganic (high Z) scintillator to act as absorbers, or a similar combination of solid state detectors (e.g. Si and CdTe). 
Computations say that the two phases configurations are much more sensitive (Costa et al. 1995), especially because of the much wider bandwidth. Yet the one-phase ones are easier to build. Arrays of high Z detectors built as a part of a system based on coded masks can be also used as a one-phase polarimeter. This has given origin to another class of polarimeters: those giving polarimetry as a byproduct of an instrument designed and built to do something else. This is very important because many of the published results of GRB polarimetry derive from this kind of experiments.

\subsection{Polarimetry of GRBs}

All the techniques described so far have been conceived for source polarimetry. Are they suited for GRBs? The simpler case is that of the Afterglow. When you point you know where it is. The polarimetry of afterglows is the same of the polarimetry of any weak source but you can integrate for a limited time. Only a photoelectric polarimeter in the focus of a large telescope is able to perform polarimetry of the brightest early afterglows. Discussing the rationale of such a measurement is not the goal of this paper, but the low level of optical polarization and the role of synchrotron suggest that the expectation of polarization could be very low. The case for a telescope dedicated to GRB polarimetry is weak, when compared with the polarimetry of other sources but the case to give fast pointing capability to any future mission of polarimetry is strong enough.

The science case for the polarimetry of the prompt is stronger but the difficulties are significantly amplified for this specific application. You do not know where the source is and need a wide field which (so far) excludes the optics. Each burst is detected at a different direction, with a different response of the instrument. You need the position and the spectrum from the same experiment or from another one. The flux may be very bright (actually it must be to perform polarimetry) and detector must be fast with dead time under control. Serious problems arise from the control of systematic effects. A pair of detectors identifies a scattering angle but the different angles are covered in a very different manner and a simple histogram is more an illustration of this coverage than of the polarization of the beam. Moreover this asymmetry strongly depends on the impact point and on the inclination of the beam with respect to the axis of the instrument. The Equation (2.2) assumes a poisson distribution of counts in the phase histogram with constant mean value. In a polarimeter devoted to sources this could be solved by rotation around the axis but in a polarimeter devoted to GRBs this is not possible because the axis direction is unknown. Various methods have been proposed to subtract these effects but no rigorous evaluation of the residual systematic effects has been worked out. Monte Carlo simulations are nowadays very good, but nothing can substitute the experimental data. The response to polarized and unpolarized beams at various energies is needed to account for all the small effects that cannot be included in the mass model, or the effects of electronics such as equalization and stability of thresholds. In the design of instruments dedicated to polarimetry, one of the drivers is the prevention of systematics. Results 
achieved with experiments that perform polarimetry as a byproduct science must be discussed with special caution.

\section{The existing measurements}

\subsection{The RHESSI data}

Coburn \& Boggs (2003) analyzed data from GRB 021206 collected with RHESSI. By studying coincident signals from pairs of Ge detectors they found, $\Pi=(80 \pm$ $20) \%$. This first detection was subsequently disconfirmed (Rutledge \& Fox 2004; Wigger 2004) since it was found that a significant fraction of pairs were not really correlated events. This shows how pairs of events, not related, can produce a modulation curve that mimics a polarization. This an important lesson for future experiments, for which these affects should be discovered by proper diagnostics and accurate calibrations before the launch, with particular attention to those experiments that were not conceived as polarimeters.

\subsection{The BATSE data}

BATSE experiment aboard GRO localized GRBs by comparing the counts of detectors oriented toward different directions. At higher energies, the calculation is complicated by the contribution of photons scattered by the different masses aboard CGRO and those reflected (backscattered) by the Earth atmosphere (this with a few ms delay). McConnell et al. (1996) hypothesized that the polarization of gamma-rays could be detected as well by this method. Following this idea Willis et al. (2005) analyzed the data of 2 strong GRBs detected by BATSE. The polarization of the GRB flux was introduced as a fit parameter. Heavy systematics are present and discussed in the paper, but it is likely that the data can be interpreted with the presence of polarization ranging in 30\%-100\% for GRB 930131 and in 40\%-100\% in GRB 960624.

\subsection{The INTEGRAL data}

In various phases of its development INTEGRAL was declared also as a polarimeter. In practice the polarimetric function was activated after many years. By analyzing coincident events on pair of Ge detectors and comparing with the distributions simulated, Kalemci et al. (2007) searched for polarization in data of GRB 041219a, the brightest ever detected by INTEGRAL, collected with the Ge Spectrometer SPI. They find a polarization of $98 \% \pm 33 \%$, that they consider more like an upper limit, because of the limited sample. By using data on radioactive sources on ground, they exclude equivalent systematics. The same data were analyzed also by McGlynn et al. (2007) that found hints of high polarization, but with very poor significance. It is worth, at this point, a short discussion about polarimetry of sources. With a similar analysis of coincidences Dean et al. (2008) found a Linear polarization $(46 \pm 10) \%$, between $0.1-1 \mathrm{MeV}$ of the Crab 
Nebula. Differently from the X-ray data the angle seems oriented with the jet. The polarization of the Crab has been also detected with IBIS, the other instrument of INTEGRAL, by analyzing the angular distribution of photons scattered from the first plane of detectors and absorbed by the second (Forot et al. 2008). The angles are compatible but the degree of polarization is very high $(>71 \%$ in the off pulse) and significantly different from that detected by SPI. Subsequently, again with IBIS data, a high polarization $(67 \pm 30 \%)$ was detected on CygX-1 above $400 \mathrm{keV}$ (Laurent et al. 2011). The analysis of SPI data on the same source at the same time, in a first moment seemed to contradict IBIS data for the flux (Jourdain et al. 2012). In a second time also SPI found the high polarization at higher energies (Jourdain et al. 2012a). A discrepancy with IBIS in the polarization angle was solved. The same analysis was performed on data collected by IBIS on GRB 041219a by Gotz et al. (2009). First they performed a separate analysis for the two main peaks. For the first peak they disconfirm the high polarization found by McGlynn et al., while they find on the second peak a polarization of $43 \pm 25 \%$. Then they divided the interval into 36 sub-intervals lasting 10 s each overlapping the nearby one of $5 \mathrm{~s}$, and searched for polarization in those where the signal in the image was stronger. In one of the interval (number 30) they find a high polarization and in a few other a certain level of polarization with different angles and with moderate significance. This suggests that polarization is fast varying during the burst. This fact, combined with the high and different impact of dead time on the two data sets can explain discrepancies between the two results. From data collected on ground with radioactive sources the authors set limits to systematic effects. A major problem is that Dean et al. in discussing their observations of the Crab, find that systematic effects, not accounted with simulations, prevail on statistical fluctuations. They divided the observation into 5 sub sets and derived an empirical statistics from the spread of results. Nothing like this was apparently applied by the other scholars that assume the poisson distribution as the basis of their analysis and, in some case, discuss the systematics as a limit to the detectable polarization but not a parameter of the estimation.

\subsection{The IKAROS/GAP data}

IKAROS (Interplanetary Kite-craft Accelerated by Radiation Of the Sun) harbors various scientific packages. One of them is The Gamma-Ray Burst Polarimeter (GAP). It is based on a block of plastic scintillator $(12 \mathrm{~cm}$ diameter and $6 \mathrm{~cm}$ thick) read with a single photomultiplier. All around the block are 12 detectors of CsI, each with its own readout photomultiplier (Yonetoku 2011). The scatterer is one block with one signal only and the line connecting its center with each absorber is assumed as the direction of the scattered photon. This reduces the modulation but makes the mass model more simple ad reliable. The instrument has been calibrated at $80 \mathrm{keV}$ with radiation of known degree of polarization normal to the instrument. The results are consistent with the simulations. With respect to a rigorous testing the calibrations at different angles and at different energies are missing. But the simple geometry of the scattering block, with one 
PMT only, and the presence of independent calibration sources for each detector, allowing for threshold equalization, make the whole adequately reliable. The main limitation is the small area resulting in a limited sensitivity, but we can hope that with more bursts arriving with time GAP is going to provide the first frame of this GRB science. In the data of the strong burst GRB 100826a GAP detected a polarization of $27 \pm 11 \%$ with a significance of $2.9 \sigma$ (Yonetoku et al. 2011a) in the assumption that the angle changes during the burst. Later GAP detected on GRB 110301a polarization of $70 \pm 22 \%$ with a statistical significance $3.7 \sigma$ and on GRB 110721a a polarization of $84+16-21 \%$ (Yonetoku et al. 2012). GAP results advocate for a high polarization of GRBs and increase the confidence on previous results.

\section{Present and future experiments}

No experiment of polarimetry of faint sources is presently approved. The most advanced is PolariS, a small satellite with two telescopes with a photoelectric detector and a scattering detector respectively (Hayashida et al. 2012). It is not clear how fast it can re-point to perform polarimetry of the early afterglow. It also includes 3 wide field polarimeters which are declared to be more sensitive than GAP. Various experiments for the polarimetry of the prompt afterglow are there. Tsubame is a small satellite of university class with a wide field instrument, a narrow field compton detector and re-pointing capability (Toizumi et al. 2011). Some of the designs proposed for polarimetry of sources have been also proposed, in a version without collimator. For a review of the scattering polarimeters see McConnell (2010). The most evolved, is POLAR a project developed by the University of Genève, to fly, in a short term, aboard the Chinese Space Station, in a joint effort with Beijing IHEP. It is based on moduli of wire like plastic scintillator $(6 \mathrm{~mm} \times 6 \mathrm{~mm} \times 200 \mathrm{~mm})$ read out with a pixel photomultiplier. The assemble of 25 moduli results into a block of $40 \times 40 \times 20 \mathrm{~cm}^{3}$ of finely subdivided plastic scintillator. This is what I call a one-phase polarimeter, all based on a single, in this case low Z, material. The long wires improve the efficiency at expenses of modulation. The most frequent interaction is compton-compton so that the energy of single photons is not determined. Ignoring the energy of the photon makes difficult to interpret the detected modulations, unless the spectrum of the GRB is known from an independent instrument. The team of POLAR has performed, beside simulations, long campaigns of testing with radioactive and synchrotron polarized and unpolarized sources. The effective threshold at $200 \mathrm{keV}$ and the strong spurious effects for off-axis detections, are the two major problems but the team is working out methods to face them (Orsi 2011). POLAR will significantly increase the sample of GAP, with some uncertainty on the spectra deconvolution, but with a better statistics. It could be the first instrument calibrated in a complete way.

A Compton telescope is itself a polarimeter, but the only one flown so far (COMPTEL) selected only forward angles and higher energies, where the modulation is negligible (Schoenfelder et al. 1993). The future Compton telescopes, 
based on finely subdivided detectors, will operate at lower energies and will accept scattering angles closer to $90^{\circ}$. Unfortunately the only one approved, aboard ASTRO-H, has a collimator that is good for sources but makes it useless for GRBs.

\section{References}

Bellazzini, R., Angelini, F., \& Baldini, L., 2004, Nucl. Instr. Meth. Phys. Res. A, 535, 477

Black, J.K., Baker, R.G., \& Deines-Jones, P., 2007, Nucl. Instr. Meth. Phys. Res. A, 581, 755

Coburn, W., \& Boggs, S.E., 2003, Nature, 423, 415

Costa, E., Cinti, M.N., Feroci, M., et al., 1995, Nucl. Instr. Meth. Phys. Res. A, 366, 161

Costa, E., Soffitta, P., Bellazzini, R., et al., 2001, Nature, 411, 662

Fabiani, S., Campana, R., Costa, E., et al., 2013, to be published in Astropart. Phys. [arXiv: $1301.1161 \mathrm{v} 1$ ]

Forot, M., Laurent, P. Grenier, I.A., et al., 2008, ApJ, 688, L29

Götz, D., Laurent, P., Lebrun, F., et al., 2009, ApJ, 695, L208

Hayashida, K., Yonetoku, D., \& Gunji, S., 2012, SPIE, 8443

Jourdain, E., Roques, J.P., \& Malzac, J., 2012, ApJ, 744, 64J

Jourdain, E., Roques, J.P., Chauvin, M., et al., 2012, ApJ, 761, 27J

Kalemci, E., Boggs, S.E., Kouveliotou, C., Finger, M., et al., 2007, ApJS, 169, 75

Krawczynski, A., Garson, Q., Guo, et al., 2011, Astropart. Phys., 34, 550

Laurent, P., Rodriguez, J., Wilms, J., et al., 2011, Science, 332, 438

Marshall, H.L., Heilmann, R., Schulz, N.S., \& Murphy, K.D., 2010, SPIE, Vol. 7732

McConnell, M., Forrest, D., Vestrand, W.T., \& Finger, M., 1996, AIP Conf. Proc., 384, 851

McConnell, M., 2010, in X-ray Polarimetry: A New Window in Astrophysics, Ronaldo Bellazzini, Enrico Costa, Giorgio Matt \& Gianpiero Tagliaferri (Cambridge University Press), 11

McGlynn, S., Clark, D.J., Dean, A.J., et al., 2007, A\&A, 466, 895

Orsi, S., Haas, D., Hajdas, W., et al., 2011, Nucl. Instr. Meth. Phys. Res. A, 648, 139

Rutledge, R.E., \& Fox, D.B., 2004, MNRAS, 350, 1288

Schoenfelder, V., Aarts, H., Bennett, K., et al., 1993, ApJS, 86, 657

Toizumi, T., Enomoto, T., Yatsu, Y., et al., 2011, Physica E, 43, 685

Weisskopf, M.C., Cohen, C.G., Kestembaum, H.L., et al., 1976, ApJ, 208, L125

Weisskopf, M.C., Elsner, R.F., Kaspi, V.M., et al., 2009: X-ray Polarimetry and Its Potential Use for Understanding Neutron Stars, ed. W. Becker, Neutron Stars and Pulsars, Ap\&SS Library (Springer-Verlag Berlin Heidelberg 2009), 357

Wigger, C., Hajdas, W., Arzner, K., Güdel, M., \& Zehnder, A., 2004, ApJ, 613, 1088

Willis, D.R., Barlow, E.J., Bird, A.J., et al., 2005, A\&A, 439, 245

Yonetoku, D., Murakami, T., Gunji, S., et al., 2011, PASJ, 63, 625

Yonetoku, D., Murakami, T., \& Gunji, S., 2011, ApJ, 743, L30

Yonetoku, D., Murakami, T., \& Gunji, S., 2012, ApJ, 758, L1 
